\title{
mTOR and ROS regulation by anethole on adipogenic differentiation in human mesenchymal stem cells
}

\author{
Yun-Hee Rhee ${ }^{1,2}$, Jeong Hwan Moon ${ }^{1,2,3}$, Ji-Hun Mo ${ }^{1,2,3}$, Tiffany Pham ${ }^{4}$ and Phil-Sang Chung ${ }^{1,2,3^{*}}$
}

\begin{abstract}
Background: Adipocyte differentiation of human mesenchymal stem cells (hMSCs) is dependent on mitochondrial metabolism and reactive oxygen species (ROS) to initiate adipocyte differentiation. Although anethole has been known as an anti-oxidant and lipid peroxidation inhibitor, there is little investigated about its role in adipogenic differentiation.

Methods: The effects on cytotoxicity and proliferation of anethole in hMSCs were measured by the MTT assay. The anti-adipogenic effect of anethole on hMSCs was analyzed by Oil Red O staining and western blot analysis. The anti-oxidant activity of anethole on hMSC was assessed by flowcytometry and fluorescence staining using 2',7' dichlorofluorescin diacetate (DCFDA). The western blotting was used to detect of phospho-Akt, phospho-mTOR, phospho-p70S6K, PPARY, and phsopho-AMP-activated kinase (AMPK).

Results: Anethole suppressed the adipogenic differentiation of hMSCs through down-regulation of Akt-mTORp70S6K-PPARY and up-regulation of AMPK. Anethole affected oxidative conditions through ROS generation. Anethole also rescued AMPK activity and reduced activation of mTOR-p70S6K-PPARY under oxidative conditions in presence of exogenous hydrogen peroxide.
\end{abstract}

Conclusion: ROS and mTOR regulation is a crucial factor in adipogenic differentiation, anethole has an important role in regulating activities of mTOR/PPARY and ROS control in adipogenic differentiation of hMSCs.

Keywords: Adipogenesis, hMSC, Anethole, ROS, AMPK, mTOR

\section{Background}

Adipogenic differentiation of human mesenchymal stem cells (hMSCs) is characterized by mitochondrial metabolism $[1,2]$. During adipogenic differentiation, autophosphorylation of insulin/insulin-like growth factor 1 (IGF-1) receptor tyrosine kinase in the presence of insulin initiates glucose transport, glucose metabolism, proadipogenic gene transcription and de novo lipid synthesis. In addition, Akt activation downstream of insulin signaling to produce the mammalian target of rapamycin (mTOR) complex further contributes to adipocyte differentiation [3-5]. Recent studies have revealed that the mTOR signaling pathway

\footnotetext{
* Correspondence: pschung@dankook.ac.kr

${ }^{1}$ Beckman Laser Institute Korea, Dankook University, 119 Dandae-ro, Cheonan 31116, Republic of Korea

${ }^{2}$ Laser Translational Clinical Trial Center, Dankook University Hospital,

Cheonan 31116, Republic of Korea

Full list of author information is available at the end of the article
}

has a critical role in the regulation of adipose tissue function [6], including adipogenesis [7], and lipid metabolism $[4,5]$. While exploring the differentiation pathway of mesenchymal stem cells, we found that mTOR has diverse functions, not only as an oncogene but also as an adipogenic inducer of peroxisome proliferater-activated receptor gamma (PPAR $\gamma$ ) through p70S6 kinase (p70S6K). In our previous study, we demonstrated that anethole had an effect on mTOR suppression [8]. Furthermore, PPAR $\gamma$ was related to the dependent signaling of cyclic adenosine monophosphate (cAMP), whose synthesis was inhibited oxidative stress [9]. When hMSCs from bone marrow were exposed to adipogenic inducing media, hMSC displayed robust lipid accumulation within 21 days [3]. In this process, basal oxygen consumption rate was coupled to the generation of ATP synthesis and intracellular ROS was increased [10]. In this regard, ROS and mTOR 
regulation may play a crucial role in the differentiation of adipocytes in mesenchymal stem cells.

Anethole, or 1-methoxy-4-(1-propenyl) benzene, is an aromatic compound (Fig. 1a) that occurs widely in nature which has been reported to have an effect to various disease, such as inflammation [11, 12], cancer [8, 13], and toxicity $[14,15]$. These biological activities are known to be attributed to anti-oxidant activity [16], lipid-peroxidation inhibition [17] and hydroxyl radical scavenging [8, 18]. These functions of anethole as an antioxidant depend on how ROS are efficiently regulated both inside and outside the cell. ROS generation is also required to initiate adipocyte differentiation and glycogen synthse kinase activation in glucose metabolism [9]. In this study, we hypothesized that anti-adipogenic effect of anethole is due to its anti-oxidant function and energy metabolism regulation through mTOR suppression. We examined whether adipogenic differentiation of hMSCs was affected by ROS and mTOR, which are regulated by anethole.

\section{Methods}

\section{Cell culture and reagents}

Human bone marrow derived mesenchymal stem cells (hMSCs, Cat\# PT-2501) were obtained from Lonza (Walkersville, MD, USA) and cultured according to the manufacturer's instructions. For adipocyte

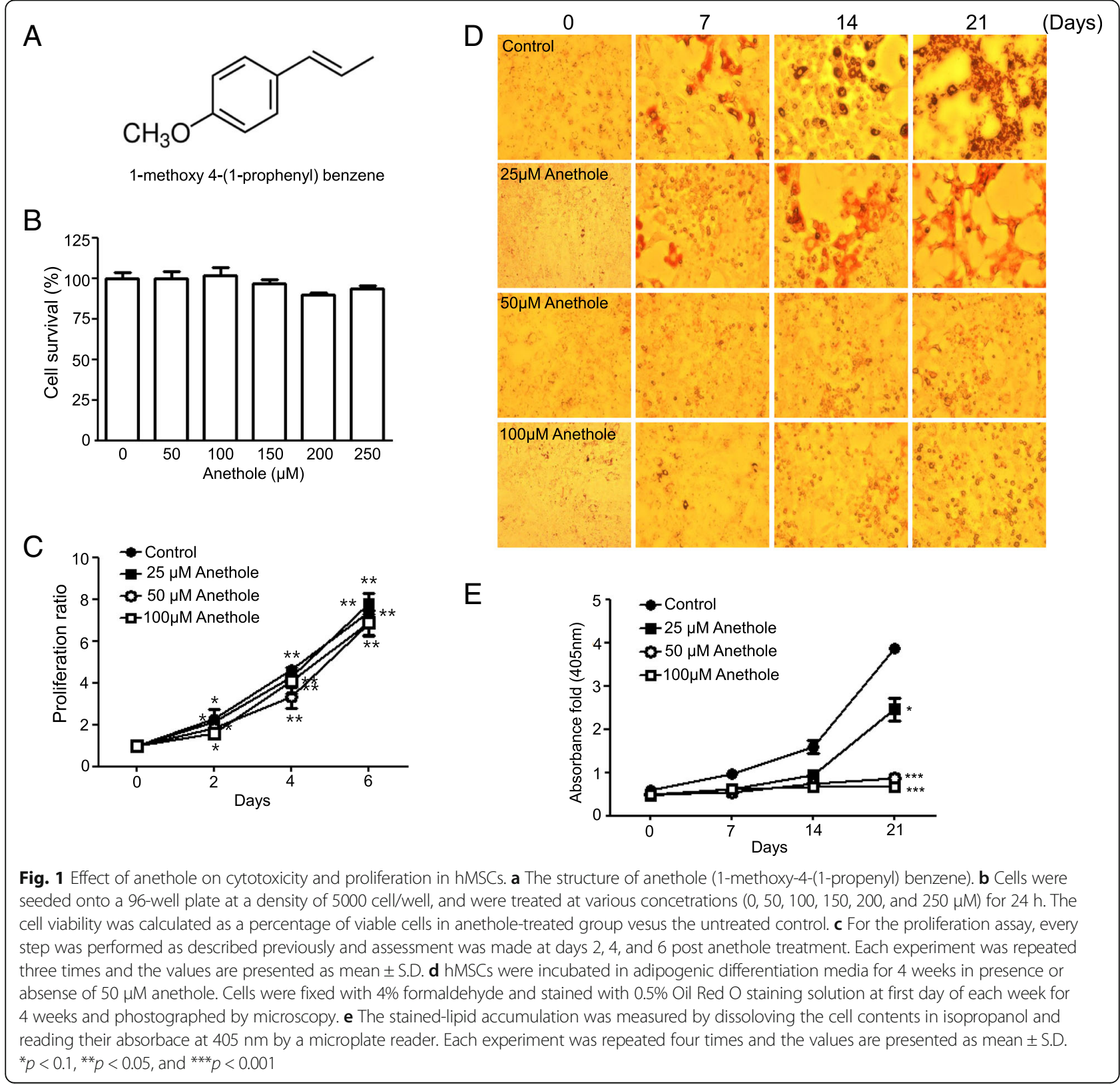


differentiation, cells were cultured for 4 weeks in high-glucose (25 mM) Dulbecco's Modified Eagles Medium (DMEM) supplemented with 10\% fetal bovine serum, $1 \%$ penicillin-streptomycin, $200 \mu \mathrm{M}$ indomethacin, $1 \mu \mathrm{M}$ dexamethasone, $10 \mu \mathrm{g}$ insulin, and $0.5 \mathrm{mM}$ isobutylmethylxanthine. DMEM, FBS, and penicillin-streptomycin were purchased from Corning (Oneonta, NY, USA). Components of adipogenic induction media and all reagents including anethole were purchased from Sigma-Aldrich (St. Louis, MO, USA).

\section{Cytotoxicity and proliferation assay}

The MTT [3-(4,5-dimethylthiazol-2-yl)-2,5-diphenyltetraxolium bromide] tetrazolium reduction assay was performed to determine the cell viability and proliferation. For cytotoxicity assessment, the cells were seeded onto a 96-well plate at a density of 5000 cells per each well. The cells were treated with anethole at various concentrations $(0,50,100,150,200$ and $250 \mu \mathrm{M})$. After $24 \mathrm{~h}, 5 \mathrm{mg} / \mathrm{ml}$ MTT was added to each well and incubated until formazan was produced. Formazan was dissolved with the MTT lysis solution (20\% SDS, 50\% dimethylformamide). The plates were measured for optical density (OD) using a microplate reader (TECAN, Männedorf, Zürich, Switzerland) at an absorbance wavelength of $450 \mathrm{~nm}$. Cell viability was calculated as a percentage of viable cells in the anethole-treated group versus the untreated control by the following equation:. Cell viability $(\%)=[\mathrm{OD}$ (anethole) - OD (Blank) / OD (Control) - OD (Blank)] × 100 . Every step was performed as previously described for proliferation assessment at days 2, 4, and 6 post anethole treatment. The proliferation ratio was calculated allowing for daily variation according to the cell viability equation. Each experiment was repeated three times.

\section{Oil red $\mathrm{O}$ staining and lipid accumulation assay}

hMSCs were incubated in adipogenic differentiation media for 4 weeks in presence or absence of $50 \mu \mathrm{M}$ anethole. Cells were then fixed with $4 \%$ paraformaldehyde and stained with $0.5 \%$ Oil Red O staining solution on the first day of each week and imaged using microscopy. After photographing the cells at 200x magnification, stained lipid accumulation was measured by dissolving the cell contents in isopropanol and reading their absorbance at $450 \mathrm{~nm}$ by a microplate reader (Biochrom, Cambridge, England).

\section{Flowcytometry analysis of ROS}

For exogenous ROS study, hMSCs were exposed at $2 \mathrm{mM}$ $\mathrm{H}_{2} \mathrm{O}_{2}$ for $30 \mathrm{~min}$ and incubated for 2 days in presence or absence of $50 \mu \mathrm{M}$ anethole. ROS were measured by staining the cells with DCFDA cellular ROS detection assay kit (Abcam, Cambridge, MA, USA) according to the manufacturer's protocol. After staining, cells were strained briefly and analyzed using Accuri-C6 (BD, Bedford, MA, USA). ROS generation was also observed under a fluorescence microscope (BX51, Olympus, Miami, FL, USA) and these samples subsequently underwent western blot analysis for p-mTOR and p-AMPK (Cell signaling, Beverly, MA, USA).

\section{Western blot analysis}

hMSCs were pretreated with $50 \mu \mathrm{M}$ anethole and incubated in adipogenic differentiation media for 3 weeks. The lysates were prepared with $100 \mu \mathrm{l}$ of lysis buffer $(50 \mathrm{mM}$ Tris- $\mathrm{HCl}$, $\mathrm{pH}$ 7.4, $300 \mathrm{mM} \mathrm{NaCl}, 0.5 \%$ Triton X-100, $5 \mathrm{mM}$ EDTA, $1 \mathrm{mM} \mathrm{Na} 3 \mathrm{VO}_{4}, 1 \mathrm{mM} \mathrm{NaF}, 10 \mu \mathrm{g} / \mathrm{ml}$ aprotinin, $10 \mu \mathrm{g} / \mathrm{ml}$ leupeptin, $10 \mu \mathrm{g} / \mathrm{ml}$ pepstatin, $10 \mathrm{mM}$ iodoacetamide, $1 \%$ phenylmethylsufonyl fluoride, PMSF) for $30 \mathrm{~min}$ on ice at every week point after treatment for 4 weeks. Total protein extracts at $30 \mu \mathrm{g}$ were separated with SDS-PAGE and electro-transferred onto a Hybond ECL membrane with the transfer buffer ( $25 \mathrm{mM}$ Tris, $250 \mathrm{mM}$ glycine, 10\% methanol). The membranes were blocked with 5\% BSA in TBST and immunoblotted for phospho-mTOR (Ser 2448), mTOR (Cell signaling), phospho-70S6K (ThermoFisher, Waltham, MA, USA), PPAR $\gamma$ (Cell signaling), phospho-Akt, Akt, phospho-AMPK, AMPK (Cell signaling) and $\beta$-actin (Sigma, St. Louis, MO, USA). After washing with TBST, the membranes were incubated with HRP-conjugated secondary antibody and developed using an ECL detection kit (GE Healthcare, Pittsburgh, PA, USA). Each protein expression was normalized by $\beta$-actin and calculated using the image J program (https://imagej.nih.gov/ij/). To study exogenous ROS, $2 \mathrm{mM} \mathrm{H}_{2} \mathrm{O}_{2}$ was added for $30 \mathrm{~min}$ at first day of adipogenic differentiation. After exposure to $\mathrm{H}_{2} \mathrm{O}_{2}$, hMSCs were incubated with adipogenic media for 2 weeks. Whole cell lysates were prepared equally at day 14 as described previously.

\section{Statistical analysis}

All data were expressed as mean \pm standard deviation (S.D.). The differences between the treatment groups and untreated controls were calculated by the Student's $t$-test one way ANOVA (Tukey test) using Prism (GraphPad, La Jolla, CA, USA). Statistical significance was determined at a value of $p^{*}<0.1, p^{* * *}<0.05$, and $p^{* * * *}<0.001$.

\section{Results \\ Effect of anethole on cytotoxicity and proliferation of hMSCs}

Anethole showed no cytotoxicity towards hMSCs up to a concentration of $250 \mu \mathrm{M}$. The cell survival rate for anethole was $100.37 \pm 9.49 \%$ at $50 \mu \mathrm{M}, 102.04 \pm 11.71 \%$ at $100 \mu \mathrm{M}, 96.78 \pm 6.2 \%$ at $150 \mu \mathrm{M}, 90.28 \pm 3 \%$ at $200 \mu \mathrm{M}$, and $93.6 \pm 4.88 \%$ at $250 \mu \mathrm{M}$. The mean survival rate at 200 and $250 \mu \mathrm{M}$ was slightly decreased, but not significantly (Fig. 1b). Anethole did not affect cell proliferation until it reached a concentration of $100 \mu \mathrm{M}$. As shown in Fig. 1c, the cell proliferation was similar in control and anethole-treated groups after 6 days. 
Effect of anethole on adipogenic differentiation in hMSCs We followed the adipogenic differentiation of hMSCs by Oil Red O staining. As the adipogenic differentiation progressed by the adipogenic inducer, the lipid accumulation was increased and with the displays of red after Oil Red O staining. As shown in Fig. 2a, hMSCs had robust lipid accumulation within 3 weeks when exposed to the adipogenic inducer cocktail of indomethacin, dexamethasone isobutylmethylxanthine (IBMX), and insulin. However, lipid accumulation was diminished (25 $\mu \mathrm{M}$ anethole) or removed (50 and $100 \mu \mathrm{M}$ anethole) after treatment with anethole. The microscope observations are shown in Fig. 1d. The quantification of lipid accumulation by measuring absorbance was verified Fig. 1e.

\section{Western blot analysis of anethole on adipogenic differentiation in hMSCs}

To investigate if adipogenic differentiation inhibition on hMSCs by anethole was dependent on the mTORPPAR $\gamma$ axis and AMPK, we performed a western blot analysis. As shown in Fig. 2, the phosphorylation of mTOR, p70S6K, and PPAR $\gamma$ rapidly increased under the induction of adipocyte differentiation and remained until 3 weeks. The phosphorylation of AMPK was reduced or unchanged with adipogenic induction of hMSCs. However, the adipogenic differentiation through activation of mTOR-p70S6K-PPAR $\gamma$ was completely inhibited by anethole treatment. The phosphorylation of mTOR decreased from the first week, whereas P70S6K and PPARy started to decrease from the second week by anethole, and remained low. Conversely, AMPK phosphorylation increased after anethole treatment, and remained high.

\section{Effect of anethole on ROS in hMSCs}

We hypothesized that ROS has a critical role on adipogenic differentiation of hMSCs. We assessed whether anethole has an effect on transcriptional programming via mTOR and AMPK by reducing excessive ROS. Hydrogen peroxide is the major form of ROS that triggers redox dependent signaling in the cytosol [10]. hMSCs were exposed at $2 \mathrm{mM} \mathrm{H}_{2} \mathrm{O}_{2}$ for $30 \mathrm{~min}$ and incubated for 2 days in the presence or absence of $50 \mu \mathrm{M}$ anethole, and subsequently stained with DCFDA. After staining, cells were analyzed by flow cytometry and fluorescence microscopy. As shown in Fig. 3a and b, ROS in hMSCs was present in some degree (18\%) in the initial stage, increasing to about $82 \%$ after the treatment with hydrogen peroxide and decreasing to about $64 \%$ in the presence of anethole. We also investigated the expression of mTOR and AMPK under oxidative stress with or without anethole by western blot analysis. As shown in Fig. 3c, ROS induced phosphorylation of mTOR and de-phosphorylation AMPK, which effects were diminished by anethole.

\section{Effect of anethole on the adipogenic markers under} excess ROS in hMSCs

To examine whether the increase in ROS affects adipogenic differentiation, hMSCs were exposed to ROS and cultured for expression of PPAR $\gamma$, a terminal adipogenic marker. As shown in Fig. 4, $\mathrm{H}_{2} \mathrm{O}_{2}$ significantly upregulated the expression of PPAR $\gamma$ and the mTOR-p70S6K signal axis and decreased expression of AMPK. However, the PPAR $\gamma /$ mTOR-p70S6K signal axis was significantly decreased in the presence of anethole, and the expression of AMPK was also restored by anethole despite the presence of $\mathrm{H}_{2} \mathrm{O}_{2}$.

\section{Discussion}

Adipogenic differentiation is a developmental process that is critical for metabolic homeostasis and nutrient signaling. mTOR kinase mediates nutrient signaling to regulate cell growth [19], proliferation [5], and diverse cellular differentiation pathways $[5,20]$. In addition, AMPK is known as a major regulator of cellular energy homeostasis and is involved in various metabolic pathways [21]. During adipogenic differentiation, AMPK and mTOR play unique roles. mTOR facilitates the accumulation of triglycerides by promoting adipogenesis and lipogenesis and by shutting down catabolic processes such as lipolysis and $\beta$-oxidation. Meanwhile, AMPK activation leads to energy preservation for cell survival at the expense of growth and proliferation via long term transcriptional control of key players of various metabolic pathways.

In this study, we assessed whether anethole could regulate mTOR and AMPK activation and could abolish excess ROS from oxidative stress during adipogenic differentiation in hMSCs. We evaluated the change of Akt-mTOR-PPAR $\gamma$ axis and AMPK phohsphorylation under oxidative stress in the presence of anethole. Although anethole has been known to not only be an anti-oxidant [16] but also a lipid-peroxidation inhibitor [17], little has been studied about its role in adipogenic differentiation. As shown in Fig. 1, cell viability and proliferation were not affected by anethole up to $100 \mu \mathrm{M}$. However, anethole inhibited the lipid accumulation of hMSCs under adipogenic inductive conditions without any changes in cytotoxicity. We investigated whether the inhibition effect on adipogenic differentiation of hMSC by anethole was dependent on the major adipogenic signaling cascade of Akt-mTOR-p70S6K-PPARy. As we expected, anethole inhibited the phosphorylation of mTOR, p70S6K, PPAR $y$ and Akt. Interestingly, the activation of AMPK was detected under presence of anethole during adipogenic differentiation of hMSCs (Fig. 2). mTOR has been reported as a major downstream effector in PI3K/Akt signaling [22], and AMPK had a contrasting activity to mTOR [2]. AMPK activation in the early phase of differentiation inhibits PPAR $\gamma$ and C/EBPs expression as well as late adipogenic markers such as fatty acid synthase (FAS) and acetyl-coA carboxylase. Several studies have revealed the convergence of AMPK and 

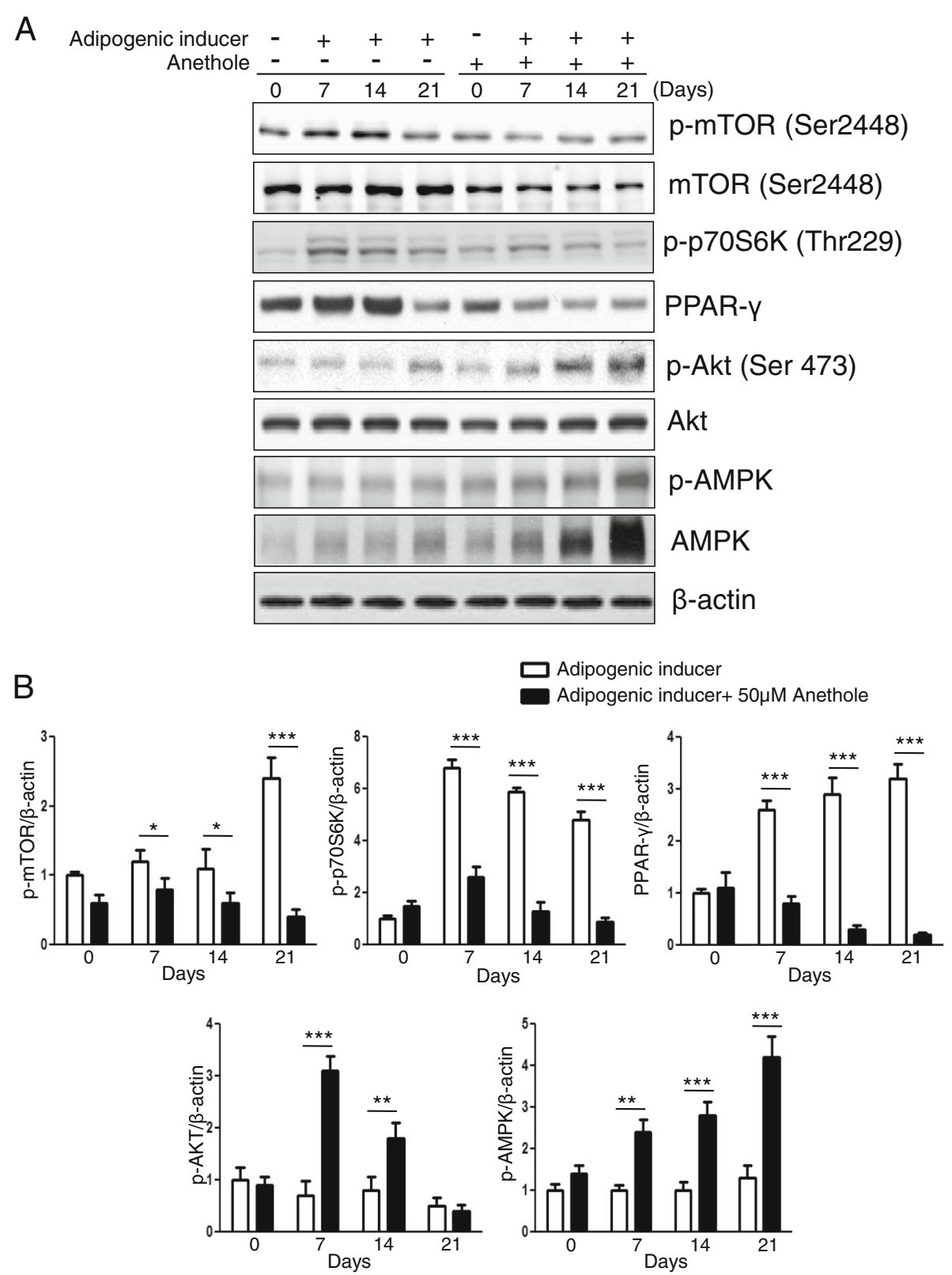

Fig. 2 Western blot analysis of anethole on adipogenic differentiation in hMSCs. a hMSCs were pretreated with $50 \mu \mathrm{M}$ anethole and incubated in adipogenic media for 4 weeks. Once a week, whole cell pellets were lysed in lysis buffer and immnoblotted for p-mTOR, mTOR, p-p70S6K, p70S6K, PPAR- $y$, p-Akt, Akt, p-AMPKand AMPK. $\mathbf{b}$ The phosphorylated expression of each protein was caculated by dividing the total form of corresponding protein, and was normalized by $\beta$-actin. The relative folds were measured and calculated using Image J. Each experiment was repeated three times and the values are presented as mean \pm S.D. ${ }^{* *} p<0.05$, and ${ }^{* *} p<0.001$

mTOR signaling pathways, pointing to mTOR as a central signal integrator that receives signals arising from growth factors, nutrients, and cellular energy metabolism [4, 23]. Mitochondrial biogenesis and metabolism are thought to be important for MSC differentiation and among them, it is presumed that the occurrence of ROS plays a crucial role in adipogenesis [24]. MSCs have low antioxidant activity and are more sensitive to oxidative stress compared to more differentiated cell types [10]. With regard to adipogenesis, ROS increases as MSCs differentiate into adipocytes, but it is unclear whether this is a cause or consequence of adipogenesis. Thus, we investigated whether excessive ROS from the addition of exogenous $\mathrm{H}_{2} \mathrm{O}_{2}$ induced the imbalance between mTOR and AMPK. We examined that $\mathrm{H}_{2} \mathrm{O}_{2}$ treatment induced excessive ROS in hMSCs by flow cytometry analysis and fluorescence microscopy observation using DCFDA (Fig. 3a and b). The phosphorylation of mTOR was elevated and the phosphorylation of AMPK was diminished after 
A

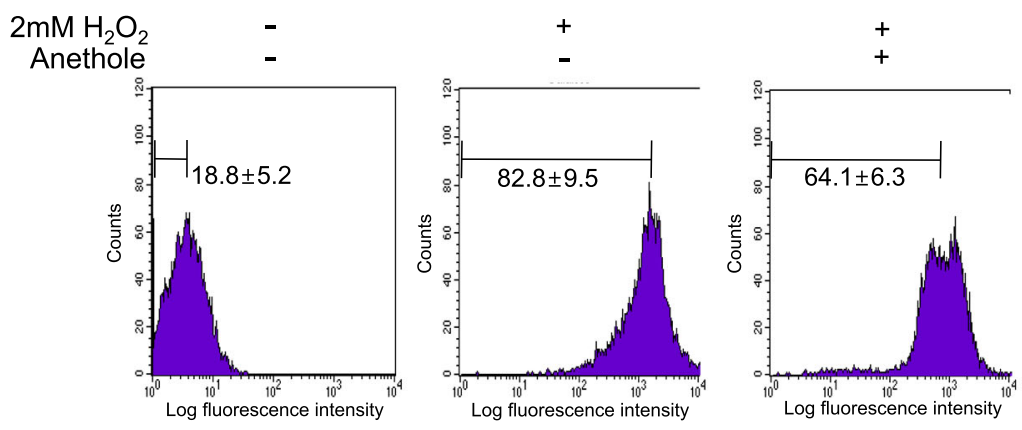

B
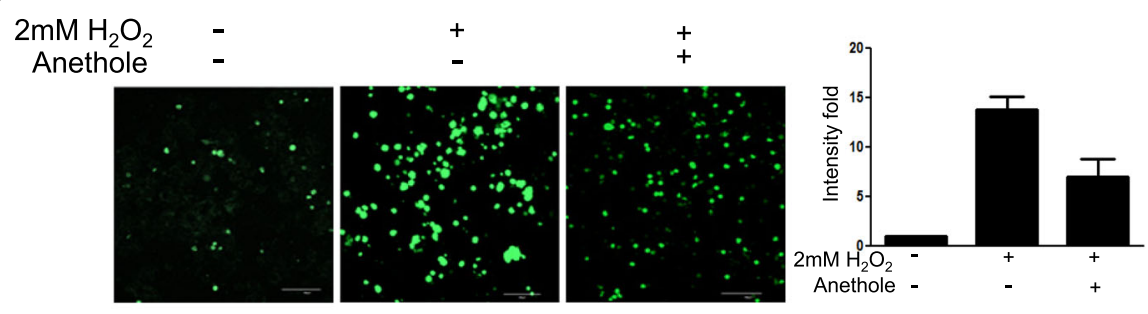

C

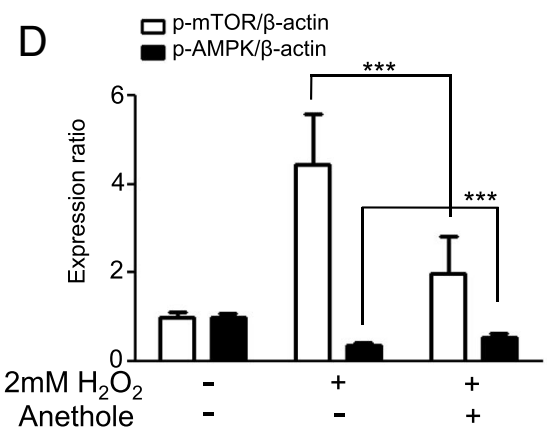

Fig. 3 Effect of anethole on excessive ROS generation in hMSCs. a hMSCs were exposed at $2 \mathrm{mM} \mathrm{H}_{2} \mathrm{O}_{2}$ for 30 min and incubated for 2 days in presence or absence of $50 \mu \mathrm{M}$ anethole. ROS was measured by staining the cells with DCFDA cellular ROS detection assay kit according to the manufacturer's instructions. After staining, cells were strained briefly and analyzed using Accuri-C6. $\mathbf{b}$ ROS generation was also observed under a fluorescence microscope at 200x magnification after same treatment previously described. c Whole cell pellets underwent western blot analysis for $\mathrm{p}$-mTOR and p-AMPK. $\mathbf{d}$ Each protein expression was exhibited in the same manner as described in Fig. 3b. Each experiment was repeated three times and the values are presented as mean \pm S.D. ${ }^{* *} p<0.05$, and ${ }^{* * *} p<0.001$

$\mathrm{H}_{2} \mathrm{O}_{2}$ treatment. However, anethole down-regulated the phosphorylation of mTOR, and up-regulated the phosphorylation of AMPK. These finding demonstrated that ROS affect the metabolic pathway between mTOR and AMPK, which could be regulated by anethole (Fig. 3c and d). Next, we assessed the expression of Akt-mTOR-PPAR signaling during adipogenic differentiation of hMSCs with and without oxidative conditions. The phosphorylation of mTORp70S6K-PPARy was accelerated only by the oxidative condition. These results demonstrated that ROS was required for activation of the transcriptional machinery for adipogenic differentiation. However, anethole regulated the over-expression of adipogenic markers and restored the AMPK activation. In particular, phosphorylation of Akt was decreased under oxidative condition without adipogenic induction, which suggests that it was relatively sensitive to oxidative stress of MSCs themselves (Fig. 4).

Several studies reported that antioxidant enzymes such as superoxide dismutase (SOD), catalase, and glutathione peroxidase (GPX) are upregulated during adipognesis in hMSCs [25], and that the ROS scavenger N-acetylcysteine (NAC) inhibits adipogenesis in the murine MSC cell line [26]. Our most significant finding indicated that ROS were crucial activators and that mTOR was a central signal integrator of cellular energy metabolism in hMSCs during adipogenic differentiation. Our results also suggested that anethole was not only an ROS scavenger but also an inhibitor of adipogenic pathways such as Akt-mTOR-PPARy. Altogether, these findings suggest anethole may provide an anti-obesity signal by regulating cellular metabolism through ROS and mTOR. 
A

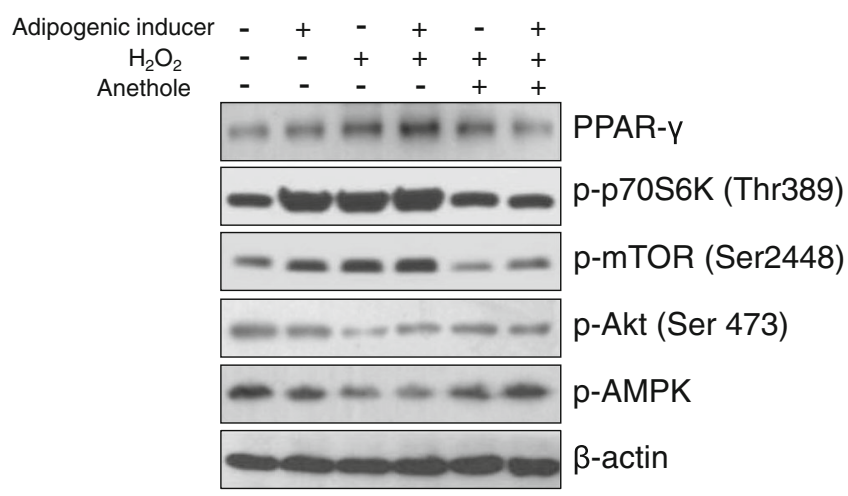

B
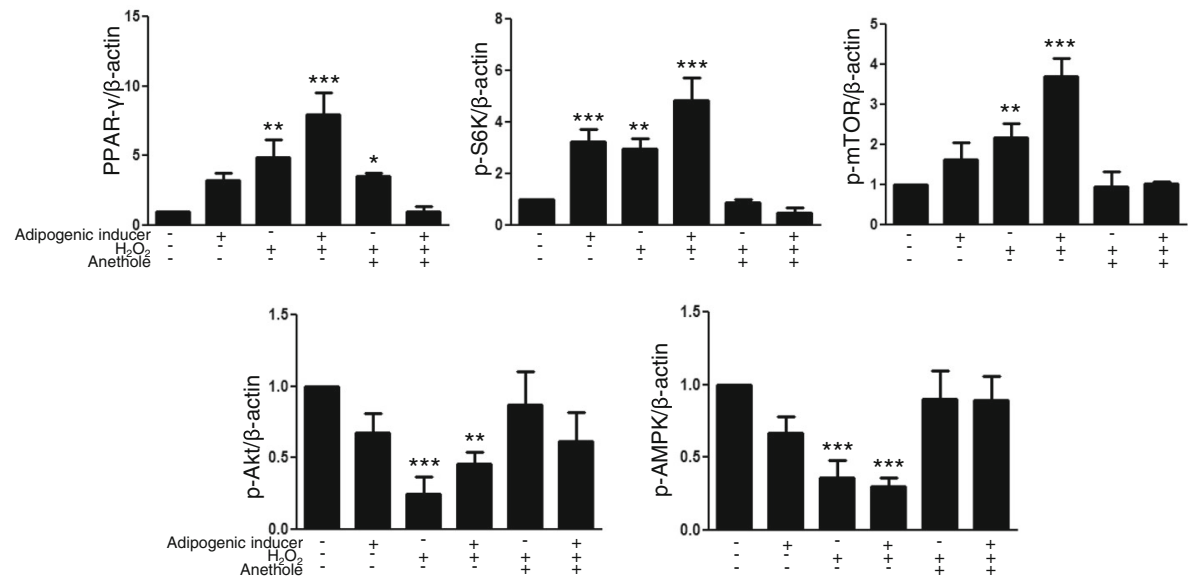

Fig. 4 Western blot analysis of theffect of anethole on transcriptional factors with excessive ROS during adipogenic differentiation. a $2 \mathrm{mM} \mathrm{H}_{2} \mathrm{O}_{2}$ was added for 30 min on the first day of adipogenic differentiation. After exposure to $\mathrm{H}_{2} \mathrm{O}_{2}$, hMSCs were incubated with adipogenic media for 2 weeks. Whole cell lysates were prepared equally at day 14 as described in Fig. 3. b Each protein expression was exhibited in the same manner as described in Fig. 3b. Each experiment was repeated three times and the values are presented as mean \pm S.D. ${ }^{* *} p<0.05$, and ${ }^{* *} p<0.001$

\section{Conclusion}

ROS and mTOR regulation is a crucial factor in adipogenic differentiation, and anethole has a potent to regulate the activities of mTOR/PPAR $\gamma$ and ROS in adipogenic differentiation of hMSCs.

\section{Additional files}

Additional file 1: The raw data of western blot. PPAR- $\gamma, \mathrm{p}-\mathrm{Akt}$, and $\beta$-actin in Fig. 2. (JPG $120 \mathrm{~kb}$ )

Additional file 2: The raw data of western blot. Akt, mTOR and AMPK in Fig. 2. (JPG $113 \mathrm{~kb}$ )

Additional file 3: The raw data of western blot. p-mTOR, p-AMPK, and p-p70S6K in Fig. 2. (JPG 114 kb)

Additional file 4: The raw data of western blot. PPAR- $\gamma, \mathrm{p}-\mathrm{AMPK}$, and and $\beta$-actin in Fig. 4. (JPG 120 kb)

Additional file 5: The raw data of western blot. p70S6K, p-mTOR, and p-Akt in Fig. 4. (JPG 106 kb)

\section{Funding}

This research was supported by Basic Science Research Program through the National Research Foundation of Korea (NRF) funded by the Ministry of Education (2017R1D1A1B03030060), the Ministry of Science and ICT (MSIT)

(2012K1A4A3053142), and by a grant of the Korea Health Technology R\&D Project through the Korea Health Industry Development Institute (KHIDI), funded by the Ministry of health \& Welfare, Republic of Korea (grant number: HI15C1524). The design of the study and material sipplements were supported by Basic Science Research Program through the National Research Foundation of Korea (NRF) funded by the Ministry of Education (2017R1D1A1B03030060), analysis and material supplements were supported by Basic Science Research Program through the National Research Foundation of Korea (NRF) funded by the Ministry of Science and ICT (MSIT) (2012K1A4A3053142), and the interpretation of data and writing editing service of this manuscript were supported by a grant of the Korea Health Technology R\&D Project through the Korea Health Industry Development Institute (KHIDI), funded by the Ministry of health \& Welfare, Republic of Korea (grant number: HI15C1524).

Availability of data and materials

All data generated or analysed during this study are included in this published article and its Additional files 1, 2, 3, 4 and 5. 


\section{Authors' contributions}

YH performed the majority of experiments, analyzed the data, and wrote the manuscript; JH (Moon) participated in animal experiments; $J H(M 0)$ and T assisted with data analysis and edited the manuscript, PS designed and coordinated the research. All authors had read and approved the manuscript, and ensure that this is the case.

\section{Ethics approval and consent to participate}

Not applicable.

\section{Consent for publication}

Not applicable.

\section{Competing interests}

The authors declare that they have no competing interests.

\section{Publisher's Note}

Springer Nature remains neutral with regard to jurisdictional claims in published maps and institutional affiliations.

\section{Author details}

'Beckman Laser Institute Korea, Dankook University, 119 Dandae-ro, Cheonan 31116, Republic of Korea. ${ }^{2}$ Laser Translational Clinical Trial Center, Dankook University Hospital, Cheonan 31116, Republic of Korea. ${ }^{3}$ Department of Otolaryngology-Head and Neck Surgery, College of Medicine, Dankook University, Cheonan 31116, Republic of Korea. ${ }^{4}$ Beckman Laser Institute and Medical Clinic, University of California, Irvine, 1002 Health Sciences Rd, Irvine, CA 92612, USA.

Received: 17 September 2017 Accepted: 25 June 2018

Published online: 06 July 2018

\section{References}

1. Yu W, Chen Z, Zhang J, Zhang L, Ke H, Huang L, Peng Y, Zhang X, Li S, Lahn BT, et al. Critical role of phosphoinositide 3-kinase cascade in adipogenesis of human mesenchymal stem cells. Mol Cell Biochem. 2008; 310(1-2):11-8.

2. Zhao L, Ha JH, Okla M, Chung S. Activation of autophagy and AMPK by gamma-tocotrienol suppresses the adipogenesis in human adipose derived stem cells. Mol Nutr Food Res. 2014;58(3):569-79.

3. Saltiel AR, Kahn CR. Insulin signalling and the regulation of glucose and lipid metabolism. Nature. 2001:414(6865):799-806.

4. Chakrabarti $P$, Kandror KV. The role of mTOR in lipid homeostasis and diabetes progression. Curr Opin Endocrinol Diabetes Obes. 2015:22(5):340-6.

5. Caron A, Richard D, Laplante M. The roles of mTOR complexes in lipid metabolism. Annu Rev Nutr. 2015:35:321-48.

6. Han J, Liang H, Tian D, Du J, Wang Q, Xi P, Wang H, Li Y. mTOR remains unchanged in diet-resistant (DR) rats despite impaired LKB1/AMPK cascade in adipose tissue. Biochem Biophys Res Commun. 2016;476(4):333-9.

7. Song BQ, Chi Y, Li X, Du WJ, Han ZB, Tian JJ, Li JJ, Chen F, Wu HH, Han LX, et al. Inhibition of notch signaling promotes the Adipogenic differentiation of mesenchymal stem cells through autophagy activation and PTEN-PI3K AKT/mTOR pathway. Cell Physiol Biochem. 2015;36(5):1991-2002.

8. Rhee YH, Chung PS, Kim SH, Ahn JC. CXCR4 and PTEN are involved in the anti-metastatic regulation of anethole in DU145 prostate cancer cells. Biochem Biophys Res Commun. 2014;447(4):557-62.

9. Rhee $\mathrm{YH}$, Ahn JC. Melatonin attenuated adipogenesis through reduction of the CCAAT/enhancer binding protein beta by regulating the glycogen synthase 3 beta in human mesenchymal stem cells. J Physiol Biochem. 2016;72(2):145-55

10. Rhee $\mathrm{S}$. Cell signaling. $\mathrm{H} 2 \mathrm{O} 2$, a necessary evil for cell signaling. Science. 2006:312:1882-3.

11. Aprotosoaie AC, Costache II, Miron A. Anethole and its role in chronic diseases. Adv Exp Med Biol. 2016:929:247-67.

12. Wisniewski-Rebecca ES, Rocha BA, Wiirzler LA, Cuman RK, VelazquezMartinez CA, Bersani-Amado CA. Synergistic effects of anethole and ibuprofen in acute inflammatory response. Chem Biol Interact. 2015;242: 247-53.

13. Chen $\mathrm{CH}$, deGraffenried LA. Anethole suppressed cell survival and induced apoptosis in human breast cancer cells independent of estrogen receptor status. Phytomedicine. 2012;19(8-9):763-7.
14. da Rocha BA, Ritter AM, Ames FQ, Goncalves OH, Leimann FV, Bracht L, Natali MR, Cuman RK, Bersani-Amado CA. Acetaminophen-induced hepatotoxicity: preventive effect of trans anethole. Biomed Pharmacother. 2017;86:213-20

15. Zahid MS, Awasthi SP, Asakura M, Chatterjee S, Hinenoya A, Faruque SM, Yamasaki S. Suppression of virulence of toxigenic Vibrio cholerae by Anethole through the cyclic AMP (CAMP)-cAMP receptor protein signaling system. PLoS One. 2015;10(9):e0137529.

16. Drukarch B, Flier J, Jongenelen CA, Andringa G, Schoffelmeer AN. The antioxidant anethole dithiolethione inhibits monoamine oxidase-B but not monoamine oxidase a activity in extracts of cultured astrocytes. J Neural Transm. 2006;113(5):593-8.

17. Stohs SJ, Lawson TA, Anderson L, Bueding E. Effects of oltipraz, BHA, ADT and cabbage on glutathione metabolism, DNA damage and lipid peroxidation in old mice. Mech Ageing Dev. 1986;37(2):137-45.

18. Tominaga H, Kobayashi Y, Goto T, Kasemura K, Nomura M. DPPH radicalscavenging effect of several phenylpropanoid compounds and their glycoside derivatives. Yakugaku Zasshi. 2005;125(4):371-5.

19. Martinez de Morentin PB, Martinez-Sanchez N, Roa J, Ferno J, Nogueiras R Tena-Sempere M, Dieguez C, Lopez M. Hypothalamic mTOR: the rookie energy sensor. Curr Mol Med. 2014;14(1):3-21.

20. Sen B, Xie Z, Case N, Thompson WR, Uzer G, Styner M, Rubin J. mTORC2 regulates mechanically induced cytoskeletal reorganization and lineage selection in marrow-derived mesenchymal stem cells. J Bone Miner Res. 2014;29(1):78-89.

21. Figarola JL, Rahbar S. Smallmolecule COH-SR4 inhibits adipocyte differentiation via AMPK activation. Int J Mol Med. 2013;31(5):1166-76.

22. Soumya SJ, Binu S, Helen A, Reddanna P, Sudhakaran PR. 15(S)-HETEinduced angiogenesis in adipose tissue is mediated through activation of PI3KJAkt/mTOR signaling pathway. Biochem Cell Biol. 2013:91(6):498-505.

23. Yue T, Yin J, Li F, Li D, Du M. High glucose induces differentiation and adipogenesis in porcine muscle satellite cells via mTOR. BMB Rep. 2010; 43(2):140-5.

24. Tormos KV, Anso E, Hamanaka RB, Eisenbart J, Joseph J, Kalyanaraman B, Chandel NS. Mitochondrial complex III ROS regulate adipocyte differentiation. Cell Metab. 2011;14(4):537-44.

25. Hiquchi M, Dusting GJ, Peshavariya H, Jiang F, Hsiao ST, Chan EC, Liu GS. Differentiation of human adipose-derived stem cells into fat involves reactive oxygen species and Forkhead box 01 mediated upregulation of antioxidant enzymes. Stem Cells Dev. 2013;22(6):878-88.

26. Kanda Y, Hinata T, Kang SW, Watanabe Y. Reactive oxygen species mediate adipocyte differentiation in mesenchymal stem cells. Life Sci. 2011;89(7-8):250-8.

\section{Ready to submit your research? Choose BMC and benefit from:}

- fast, convenient online submission

- thorough peer review by experienced researchers in your field

- rapid publication on acceptance

- support for research data, including large and complex data types

- gold Open Access which fosters wider collaboration and increased citations

- maximum visibility for your research: over $100 \mathrm{M}$ website views per year

At BMC, research is always in progress.

Learn more biomedcentral.com/submissions 